\title{
Diaphragm disease and chronic NSAID use
}

\begin{abstract}
NSAIDs' induced inflammation and ulceration in the upper GIT tract and small intestine is well described in the literature, while its effect on the colon is less known by the physicians. NSAIDs' enteropathy can present with a wide clinical presentation varying from anemia, abdominal distension to pain and obstruction. Endoscopic features will show colonic ulcerations, erythema, or diaphragmatic mucosal folds with constrictions and obstruction. Management includes withdrawal of the drug, balloon dilatation and may reach to hemicolectomy. Herein, we present a case of diaphragmatic colonic constrictions related to the chronic use of non-steroidal anti-inflammatory drug (NSAID).
\end{abstract}

Keywords: diaphragm disease, colonoscopy, NSAIDS, colopathy, erythema
Volume 10 Issue 4 - 2019

\author{
Mahmoud Othman \\ Department of Internal Medicine \& Gastroenterologist, Nini \\ Hospital, Lebanon
}

Correspondence: Mahmoud Othman, Department of Internal
Medicine \& Gastroenterologist, Nini Hospital Nini Hospital, Lebanon,Tel 0096I0354565I,Email mm_1983@yahoo.com

Received: August 12,2019 | Published: August 27, 2019
Abbreviations: NSAID, non-steroidal anti-inflammatory drugs; $\mathrm{CT}$, computed tomography; EGD, esophagogastroduodenoscopy; $\mathrm{DD}$, diaphragm disease

\section{Introduction}

NSAIDs have been prescribed widely by the physicians for the management of many diseases and patient's complains. Although, the adverse effects of NSAIDs on the upper GI tract are well known, lower GIT side effects are less described in the literature. NSAID induced ceacal ulceration was first described in $1966 .{ }^{1}$ NSAID induced colopathy has wide presentation mimicking malignancy or IBD. Physicians must be aware in their differentials of anemia with a history of chronic NSAID use to the lower as Upper GIT bleed and obstruction. Diaphragm disease is one of the presentations of NSAID colopathy, characterized by concentric stenotic lesions with obstruction. Herein, we present a case report of diaphragm disease which was managed by endoscopic dilatation with a review of literature of this uncommon NSAID side effect.

\section{Case presentation}

A 54-year-old female known to have osteoarthritis on diclofenac since 1year and DVT on Coumadin present to our hospital complaining of recurrent bouts of abdominal pain, distention and fatigue of few months' duration. Physical exam revealed abdominal tympani and mild direct tenderness but was otherwise normal. Workup revealed anemia (hemoglobin $=5.2 \mathrm{mg} / \mathrm{dl}$ ); basic metabolic panel, liver function tests, amylase/lipase, and urine analysis were all within normal limits. Computed Tomography (CT) of the abdomen showed patchy colonic wall thickening. Esophagogastroduodenoscopy (EGD) was normal, while colonoscopy revealed multiple colonic strictures in the descending colon (Figures 1A\&1C) with surrounding edema and erythema (Figures 1B\&1D). The pediatric colonoscopy could not be advanced beyond the hepatic flexure due to severe stricturing. Patient was diagnosed having NSAID colopathy with Diaphragm disease. She was treated with discontinuation of diclofenac and endoscopic balloon dilatation to relieve obstruction (Figures 1B\&1D). The patient consequently had relief of symptoms and refused a repeat colonoscopy to dilate more proximal strictures.
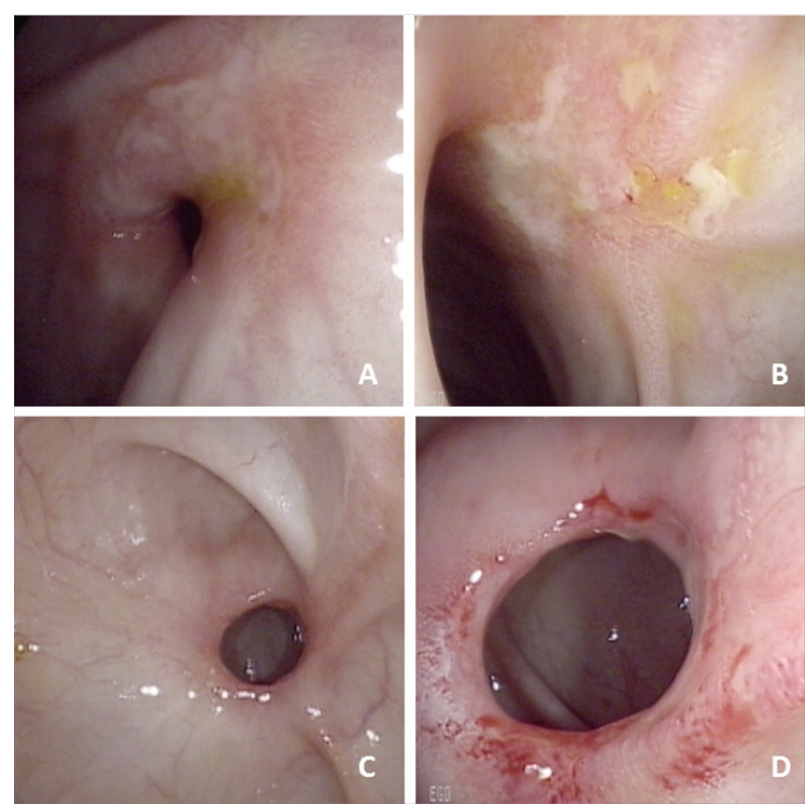

Figure I Colonoscopic figures showing edema and ulceration (A \& B), stenosis (C) and erythema (D) in the descending colon.

\section{Discussion}

Diaphragm disease is a rare GIT tract that was found to be related to chronic NSAIDs use. It is characterized by the development of multiple concentric protrusions of fibrotic mucosa nearly occluding the lumen. DD is one of the NSAIDs side effects at the lower GIT where it was first coined by Lang el al. ${ }^{2}$ who presents 7 cases with $\mathrm{DD}$ at the small intestine exclusively. Colonic diaphragmatic disease was first described by Sheers and Williams in 1989. ${ }^{3}$ The presence of diaphragms in the colon has been found to be pathognomonic for NSAIDs related injury with around to 48 cases described to date. ${ }^{4}$ It's more common in elderly with mean age of 65 . Females have more risk which was related to the more incidence of musculoskeletal and rheumatic disorders. ${ }^{5}$

The pathophysiology of the disease is still unclear, where some correlates it with micro vascular injury leading to bile, bacteria acting 
as chemoattractant and affecting the mucosa. Others suggest that it is related to antibodies to cyclooxygenase. ${ }^{6}$ Many NSAIDs have been associated with diaphragm formation, but mostly followed the introduction of extended-release NSAIDs which support the hypothesis that correlates NSAID colopathy with local side effect. Right colon is the most affected part of the large intestine.

Patients with DD complain of nonspecific mostly chronic symptoms of anemia, obstruction, and altered bowel habits. Acute presentation is rare with one case report presenting with perforated colon. ${ }^{7}$ Differentials are colorectal cancer, IBD, IBS and colitis. Diagnosis is based on endoscopic findings of diaphragmatic lesions which may leads to perform a full examination of both the small and large intestines, including an intraoperative enteroscopy due to multiple lesions.

Management is unclear in the literature. Data suggest discontinuation of NSAIDs with either endoscopic dilatation or surgical resection with only 22 case reports treated by dilatation ().Some publication suggests the role of steroid therapy in refractory cases. $^{8}$ Others suggest usage of amino salicylic acid (5 ASA). Patients should be advised not to resume NSAID whenever possible for the recurrence of the strictures in several reported cases in the literature. ${ }^{10-12}$

\section{Conclusion}

NSAIDs have great side effects on lower GIT as upper part. Chronic use of NSAIDs may leads to colonic ulceration, bleed, obstruction, and even perforation. Diaphragm disease is multiple strictures with concentric fibrotic mucosa leading to obstruction and anemia.DD is found to be pathognomic for chronic use of NSAIDs. It may occur in small intestine and less reported cases in the large intestine. NSAIDs should be stopped and either endoscopic dilatation or surgical resection should be done according to the severity, number of lesions or recurrence.

\section{Acknowledgments}

None.

\section{Conflicts of interest}

The authors declare that there is no conflict of interest.

\section{Funding}

None.

\section{References}

1. Debenham GP. Ulcer of the cecum during oxyphenbutazone (tandearil) therapy. Can Med Assoc J. 1966;94(22):1182-1184.

2. Lang J, Price AB, Levi AJ, et al. Diaphragm disease: pathology of disease of the small intestine induced by non-steroidal anti-inflammatory drugs. J Clin Pathol. 1988;41(5):516-526.

3. Sheers R, Williams WR. NSAIDs and gut damage. Lancet. 1989;2(8672):1154.

4. Wang YZ, Sun G, Cai FC, et al. Clinical Features, Diagnosis, and Treatment Strategies of Gastrointestinal Diaphragm Disease Associated with Nonsteroidal Anti-Inflammatory Drugs. Gastroenterol Res Pract. 2016:3679741.

5. Zhao B, Sanati S, Eltorky M. Diaphragm disease: complete small bowel obstruction after long-term nonsteroidal anti-inflammatory drugs use: a case report and review of literature. Ann Diagn Pathol. 2005;9(3):169173.

6. Bjarnson I, Hayllar J, Macpherson AJ, et al. Side effects of nonsteroidal anti-inflammatory drugs on the small and large intestine in humans. Gastroenterology. 1993;104(6):1832-1847.

7. Robinson MH, Wheatley T, Leach IH. Nonsteroidal antiinflammatory drug-induced colonic stricture - an unusual cause of large bowel obstruction and perforation. Dig Dis Sci. 1995;40(2):315-319.

8. Weinstock LB, Hamoud Z, Brandwin L. Nonsteroidal anti-inflammatory drug-induced colonic stricture and ulceration treated with balloon dilatation and prednisone. Gastrointest Endosc. 1999;50(4):564-565.

9. Penner RM, Williams CN. Resolution of multiple severe nonsteroidal anti-inflammatory drug-induced colonic strictures with prednisone therapy: a case report and review of the literature. Can J Gastroenterol. 2003;17(8):497-500.

10. Monahan DW, Starnes EC, Parker AL. Colonic strictures in a patient on non-steroidal anti-inflammatory drugs.Gastrointest Endosc. 1992;38(4):385-388.

11. Eis MJ, Watkins BM, Philip A, et al. Nonsteroidal-induced benign strictures of the colon: A case report and review of the literature.Am J Gastroenterol. 1998;93(1):120-121.

12. Spirnak JP. Colonic diaphragms associated with long-term use of nonsteroid antiinflammatory drugs. Am J Radiol. 1993;160:1148-1149. 